\title{
One-dimensional dynamics of nearly unstable axisymmetric liquid bridges
}

\author{
José M. Perales and José M. Vega \\ E.T.S.I. Aeronáuticos, Universidad Politécnica de Madrid, 28040 Madrid, Spain
}

\begin{abstract}
A general one-dimensional model is considered that describes the dynamics of slender, axisymmetric, noncylindrical liquid bridges between two equal disks. Such model depends on two adjustable parameters and includes as particular cases the standard Lee and Cosserat models. For slender liquid bridges, the model provides sufficiently accurate results and involves much easier and faster calculations than the full three-dimensional model. In particular, viscous effects are easily accounted for. The one-dimensional model is used to derive a simple weakly nonlinear description of the dynamics near the instability limit. Small perturbations of marginal instability conditions are also considered that account for volume perturbations, nonequality of the supporting disks, and axial gravity. The analysis shows that the dynamics breaks the reflection symmetry on the midplane between the supporting disks. The weakly nonlinear evolution of the amplitude of the perturbation is given by a Duffing equation, whose coefficients are calculated in terms of the slenderness as a part of the analysis and exhibit a weak dependence on the adjustable parameters of the one-dimensional model. The amplitude equation is used to make quantitative predictions of both the (first stage of) breakage for unstable configurations and the (slow) dynamics for stable configurations.
\end{abstract}

\section{INTRODUCTION}

A liquid bridge is the configuration that appears when a liquid mass is held by capillary forces between two solid supports. The configuration offers the simplest mechanical model for some complex systems that appear in many phenomena and is of industrial interest in, e.g., the crystal growth technique known as floating zone.

In the past, one can find many papers dealing with the statics of liquid bridges. A review can be found in Meseguer et al. ${ }^{1}$ The previous works focused mainly on determining the parameter values for which the liquid bridge is either stable or unstable. The instability (breakage) for axisymmetric liquid bridges can have two very different behaviors. For slender (long) liquid bridges the configuration breaks into two drops of different volumes, while for shorter bridges the two drops are equal. In both cases, after the breakage, a third much smaller drop (the so-called satellite) appears in between. For even shorter liquid bridges, the instability is nonaxisymmetric and leads no longer to a breakage but to a nonaxisymmetric deformation that can be recovered by simply returning the values of the parameters to their original values.

Cylindrical liquid bridges were considered in the pioneering analysis by Rayleigh, ${ }^{2}$ who found that these liquid bridges destabilize when their length is greater than its circumference. More recent stability analyses have focused on the effect of various, not necessarily small perturbations, such as axial gravity/acceleration, ${ }^{3}$ unequal disks diameter, ${ }^{4}$ rigid solid rotation, ${ }^{5}$ and the combined effect of the above. ${ }^{6,7}$ In all these, although the basic configurations were axisymmetric, the possible instabilities were allowed to be either axisymmetric or nonaxisymmetric.
Additional studies cover the influence of various nonaxisymmetric perturbations. ${ }^{8,9}$ The weakly nonlinear analysis for small perturbations is not easy since it often requires an asymptotic analysis to clarify the effect of the various, qualitatively different perturbations. The case of nearly cylindrical liquid bridges was considered by Vega and Perales. ${ }^{10}$ An extension of this analysis to liquid bridges of general form has been more recently made, focusing on the influence of small perturbations in the change of stability of liquid bridges close to the stability limit. ${ }^{11}$

The theoretical predictions mentioned above have been corroborated by experiments, either on board of space laboratories (where residual gravity is much smaller) or on ground; in the latter case, both Plateau baths and microzones ${ }^{12-14}$ have been used.

A considerable amount of theoretical, ${ }^{15-25}$ numerical, ${ }^{26-29}$ or experimental ${ }^{30-32}$ works in the literature deal with linear vibrations. A less understood problem is connected with the dynamics near the stability limit. Available results are really scarce and either consider only the vicinity of the point where a cylindrical liquid bridge loses stability (the Rayleigh limit; see Sanz, ${ }^{33}$ Higuera et al., ${ }^{34,35}$ and Nicolás and $\mathrm{Vega}^{36}$ ) or are based on one-dimensional approximations, such as the so-called Cosserat model. ${ }^{37-41}$

Although the study of the dynamics of near-unstable, noncylindrical liquid bridges using an inviscid (potential) three-dimensional axisymmetric model may be done, the present paper relies on a simpler one-dimensional (1D) model, which both involves much simpler calculations and allows for the straightforward inclusion of viscous damping. A pioneer work using 1D models for analyzing the dynamics was done by Rivas and Meseguer. ${ }^{37}$ In this work, a particular case was considered and the analysis was restricted to nearly cylindrical liquid bridges between equal disks $\left(\Lambda=\pi, V_{0}=1\right.$, 


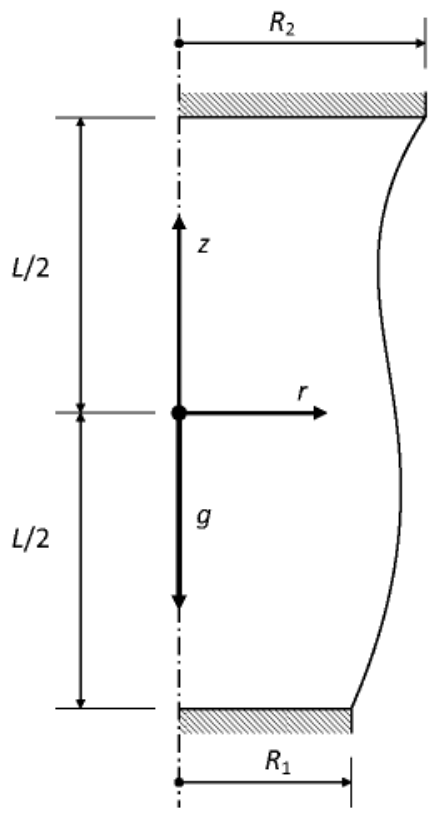

FIG. 1. A meridian section of the liquid bridge configuration.

$C \ll 1,|V| \ll 1,|H|=0,|B| \ll 1$, see below for the meaning of the different parameters. The present work extends those results to the neighborhood of the stability limit in a wide interval.

The geometry and the various parameters involved are sketched in Fig. 1. In the following, all expressions are made dimensionless using capillary time $\left(\rho R_{0}{ }^{3} / \sigma\right)^{1 / 2}$ and the mean of the (almost equal) disk radii $R_{0}=\left(R_{1}+R_{2}\right) / 2$ to nondimensionalize time and length, respectively, and the ratios $\left(\sigma / \rho R_{0}\right)^{1 / 2}$ and $\sigma / R_{0}$ as velocity and pressure units, respectively. The basic parameters defining the configuration are the slenderness $\Lambda=L /\left(2 R_{0}\right)$ and the dimensionless volume $V /\left(\pi L R_{0}^{2}\right)$. The analysis is made in the vicinity of the instability limit, which corresponds to a nondimensional volume $V_{0}$, which depends on $\Lambda$; small perturbations of the nondimensional volume $V=\boldsymbol{V} /\left(\pi L R_{0}{ }^{2}\right)-V_{0}$ will be considered as well as small values of the dimensionless disk radii difference $H=\left(R_{2}-R_{1}\right) /\left(R_{2}+R_{1}\right)$ and small values of the Bond number $B=\rho g R_{0}{ }^{2} / \sigma$. Viscous effects are measured by the Ohnesorge number $C=\mu / \sqrt{\rho \sigma R_{0}}=v / \sqrt{\sigma R_{0} / \rho}$, which is assumed to be small. Here, $\rho$ is the density, $\sigma$ the surface tension, and $\nu$ the kinematic viscosity. Namely, we assume that

$\Lambda \sim 1, \quad V_{0} \sim 1, \quad C \ll 1, \quad|V| \ll 1, \quad|H| \ll 1, \quad|B| \ll 1$.

\section{PROBLEM FORMULATION, STEADY STATES, AND INSTABILITY LIMITS}

Restricting to axisymmetric configurations, onedimensional models for the dynamics assume a functional form for the radial variation of the axial velocity; the simplest assumption is that the axial velocity is constant in each cross section (Lee model). Introducing this functional form into the continuity and radial momentum equations, two functional forms result for the radial variation of pressure and the radial velocity; the latter is slave to the axial velocity variation along the axial coordinate and depends linearly on the radial coordinate in the simplest Lee model. More complex variations may be considered yielding to more accurate models, such as the Cosserat and the averaged models. A further substitution into the axial momentum conservation equation and averaging in the radial direction yields ${ }^{38,39}$

$$
\begin{aligned}
W_{t}+ & W W_{z}+\left(k_{1}+k_{2}\right)\left[-\frac{1}{2} F F_{z}\left(W_{t z}-\frac{1}{2} W_{z}^{2}+W W_{z z}\right)\right. \\
& \left.-\frac{1}{8} F^{2}\left(W_{t z}-\frac{1}{2} W_{z}^{2}+W W_{z z}\right)\right] \\
= & -P_{z}-B+C\left[3 W_{z z}+6 \frac{F_{z}}{F} W_{z}\right] \\
& +C k_{1}\left[-\frac{1}{8} F^{2} W_{z z z z}-F F_{z} W_{z z z}-\frac{1}{2}\left(F F_{z z}+3 F_{z}^{2}\right) W_{z z}\right] \\
& +\frac{3}{4} C k_{2}\left[\left(F F_{z z}-3 F_{z}^{2}\right) W_{z z z}\right. \\
& \left.+\left(F F_{z z z}-3 F_{z} F_{z z}-6 \frac{F_{z}}{F} F_{z}^{2}\right) W_{z z}\right]
\end{aligned}
$$

where $W(z, t)$ is the radially averaged axial velocity, $F(z, t)$ the shape of the liquid bridge interface [defined as $r=F(z, t)], P(z, t)$ the pressure at the interface, and subscripts denote hereafter derivation with respect to $t, r$, and $z$. Varying the parameters $k_{1}$ and $k_{2}$, three different one-dimensional models are obtained, namely, the Lee model with $k_{1}=0$, $k_{2}=0$, the Cosserat model with $k_{1}=1, k_{2}=0$, and the averaged model with $k_{1}=0, k_{2}=1$. Note that viscous effects are straightforwardly included in those terms proportional to $C$, which will account below for viscous dissipation in the bulk. Viscous dissipation in the boundary layers attached to the disks instead will be ignored (imposing a free stress boundary condition at the disks, see below), which is a good assumption already in the three dimensional case (see Refs. 35, 36 , and 42 for the validity of this assumption in the liquid bridge geometry and in cylindrical containers under both axisymmetric and nonaxisymmetric perturbations).

Equation (2) involves three unknowns and thus two additional equations are necessary, which result from kinematic compatibility (related to the continuity equation) and equilibrium of normal stress at the free surface, namely,

$$
\begin{aligned}
& F_{t}(z, t)=-\frac{1}{2} F(z, t) W_{z}(z, t)-F_{z}(z, t) W(z, t), \\
& P(z, t)+\mathrm{M}[F(z, t)]=0,
\end{aligned}
$$

where $\mathrm{M}[F]$ is the mean curvature of the free surface, which is given by

$$
\mathrm{M}[F]=\frac{F F_{z z}-1-F_{z}^{2}}{F\left(1+F_{z}^{2}\right)^{3 / 2}} .
$$

The boundary conditions for $W$ and $F$ are

$$
W( \pm \Lambda, t)=0,
$$




$$
\begin{aligned}
& F( \pm \Lambda, t)=1 \pm H, \\
& W_{z}( \pm \Lambda, t)=0,
\end{aligned}
$$

where the first two account for disk impenetrability and free surface anchoring to the sharp disk edges and the latter can be deduced from Eq. (3).

For convenience, we also state volume conservation

$$
\int_{-\Lambda}^{\Lambda} F^{2}(z, t) \mathrm{d} z=2 \Lambda\left(V_{0}+V\right) .
$$

Finally, the initial conditions for the velocity field $W(z, 0)$ and the interface shape $F(z, 0)$ must be compatible both with Eqs. (3) and (4) and the boundary conditions (6)-(9) and with the perturbation scheme used below.

The quiescent steady states are such that $W=0, P=P_{0}$ $=$ const. Thus, the associated free surface deformation is given by the nonlinear ordinary differential equation (4), which now reads $\mathrm{M}\left[F_{0}\right]+P_{0}=0$ and determines $F_{0}$ when the boundary conditions ( 7$)$ and (9) are taken into account. The instability limit of these is readily calculated imposing that the linearized problem around the steady states exhibits a nontrivial solution (see, e.g., Martínez and Perales ${ }^{43}$ ). Namely, the perturbation of the free surface $f=F-F_{0}$ is given by the following linearized, second order, two-point boundary value problem:

$$
\mathbf{M}^{\prime}\left[F_{0}\right][f]+p=0, \quad f( \pm \Lambda)=0,
$$

where the linear operator $\mathrm{M}^{\prime}\left[F_{0}\right]$ is the Frechet derivative of the mean curvature operator (5), namely,

$$
\begin{aligned}
\mathrm{M}^{\prime}\left[F_{0}\right][f]= & \frac{1}{\left(1+F_{0 z}^{2}\right)^{3 / 2}} f_{z z} \\
& +\left[\frac{F_{0 z}}{F_{0}\left(1+F_{0 z}^{2}\right)^{3 / 2}}-\frac{3 F_{0 z} F_{0 z z}}{\left(1+F_{0 z}^{2}\right)^{5 / 2}}\right] f_{z} \\
& +\frac{1}{F_{0}^{2}\left(1+F_{0 z}^{2}\right)^{1 / 2}} f \\
= & \frac{1}{F_{0}} \frac{d}{d z}\left[\frac{F_{0} f_{z}}{\left(1+F_{0 z}^{2}\right)^{3 / 2}}\right]+\frac{f}{F_{0}{ }^{2}\left(1+F_{0 z}^{2}\right)^{1 / 2}} .
\end{aligned}
$$

Hereinafter, in all Frechet derivatives, the first square brackets pair contains the point where the operator is evaluated and the second square brackets pair contains the function to which the operator is applied. For each value of the slenderness, this condition (and its counterpart considering also nonaxisymmetric perturbations) provides two (lower and upper) threshold values of the volume, which will be called the instability limits below. These are plotted for $B=H=0$ in Fig. 2 , where the stable configurations are those in between the instability limits. The upper instability limit is associated with nonaxisymmetric perturbations. The lower instability limit instead is associated with axisymmetric perturbations, which are either reflection symmetric on the plane $z=0$ if $\Lambda_{A} \cong 2.127>\Lambda>\Lambda_{C} \cong 0.361$ or antisymmetric in $z$ if $\Lambda$ $>\Lambda_{A} \cong 2.127$. Thus, in the former case, the breaking process is essentially symmetric with regard to the midplane, giving one smaller central drop and two larger lateral drops, while

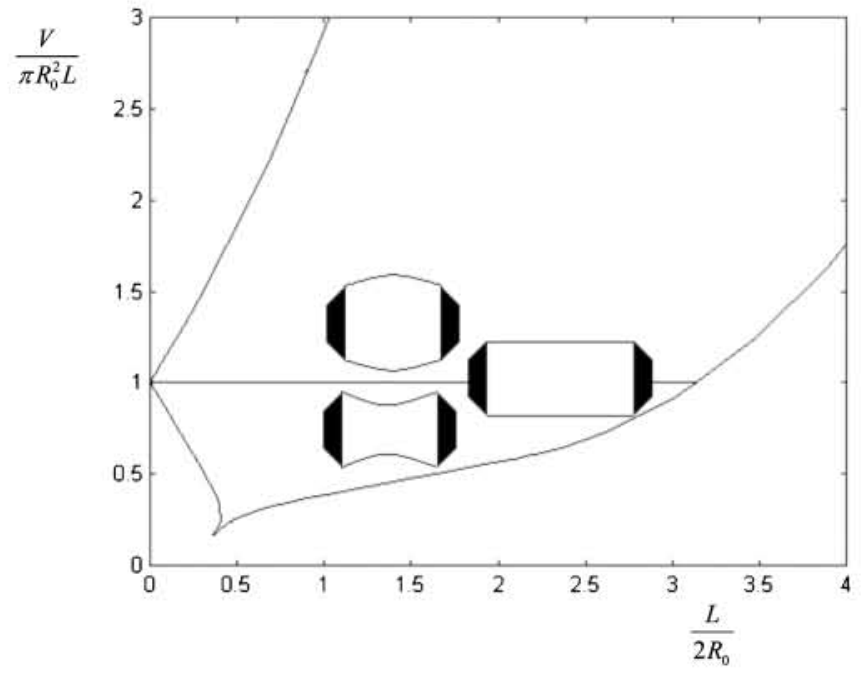

FIG. 2. Stability limits of liquid bridges between equal disks for zero Bond number. Sketches show stable liquid bridges with volumes larger, equal, and smaller than those corresponding to a cylinder.

in the latter case, the liquid bridge breaks into two clearly different drops (with a third smaller drop in between). If $\Lambda<\Lambda_{C}$, the unstable perturbations are again nonaxisymmetric, which are outside the scope of this paper. Note that the symmetric and antisymmetric instability limits are tangent to each other at $\Lambda=\Lambda_{A}$, where a mode interaction process associated with a codimension 2 bifurcation takes place, whose analysis is again outside of the scope of this paper.

Against this background, the main object of this paper is to describe both oscillations and breaking near the instability limits in a weakly nonlinear setting, namely, assuming that perturbations of the free surface deflection are small. Since a one-dimensional model of the dynamics is used, only the case of slender liquid bridges $\Lambda>\Lambda_{A}$ will be considered. For the sake of clarity, we anticipate here the main results.

In the nonsymmetric case (near the lower instability limit in Fig. 2 as $\Lambda>\Lambda_{A}$ ), perturbations of the free surface are given by

$$
F(z, t)=F_{0}(z)+A(t) f(z)+\cdots,
$$

where $f(z) \sim 1$ is a solution of the linearized problem (10) and the mode amplitude $A(t)$ is small, namely,

$$
|A(t)| \ll 1,
$$

and given by the following Duffing equation:

$$
\mu \frac{\mathrm{d}^{2} A(t)}{\mathrm{d} t^{2}}+\gamma C \frac{\mathrm{d} A(t)}{\mathrm{d} t}+\widetilde{g}_{12} V A(t)+\widetilde{g}_{111} A^{3}(t)=\widetilde{g}_{3} H+\widetilde{g}_{4} B .
$$

In the absence of both forcing and damping $(C=H=B=0)$, this equation generally gives perturbations of one mode oscillations in conservative systems in the presence of reflection symmetry, and has been widely studied; see Nayfeh and Mook, ${ }^{44}$ Guckenheimer and Holmes, ${ }^{45}$ Moon, ${ }^{46}$ and Thompson and Stewart ${ }^{47}$ for comprehensive reviews of the associated literature. Here, the dependence of the various coefficients on the small parameters $C, V, H$, and $B$ and the fact 
that nonlinearity is cubic are anticipated from symmetry considerations, noting that the equation must be invariant under the symmetry group

$$
A \rightarrow-A, H \rightarrow-H, B \rightarrow-B
$$

Assumptions (1) and (13) imply that the amplitude $A(t)$ evolves in a large timescale (compared with the capillary timescale used above for nondimensionalization). Equation (14) allows a complete understanding of the dynamics near the instability limit. The derivation of this equation (and the calculation of the numerical values of the coefficients in terms of the slenderness) is now performed as the main object of this paper.

\section{DERIVATION OF THE AMPLITUDE EQUATION}

If $\Lambda>\Lambda_{A}$ (with $\Lambda_{A}=2.127 \cdots$, see Martínez and Perales ${ }^{43}$ ), an order of magnitude analysis leads the appropriate scaling for both the parameters,

$$
\begin{aligned}
& V=\varepsilon^{2} v, \quad B=\varepsilon^{3} b, \quad H=\varepsilon^{3} h, \\
& \tau=\varepsilon t, \quad C=\varepsilon c,
\end{aligned}
$$

and the state variables, where for the sake of clarity, $\varepsilon \ll 1$ is the only small parameter that will be considered, namely, it will be assumed that $v \sim b \sim h \sim c \sim \tau \sim 1$. The state variables are seen to be such that $U \sim W \sim P-P_{0} \sim F-F_{0} \sim \varepsilon$, where $P_{0}$ and $F_{0}$ account for the steady state and are given by

$$
\begin{aligned}
& \mathrm{M}\left[F_{0}(z)\right]+P_{0}=0, \\
& F_{0}( \pm \Lambda)=1, \\
& \int_{-\Lambda}^{\Lambda} F_{0}^{2}(z) \mathrm{d} z=2 \Lambda V_{0} .
\end{aligned}
$$

The solution to this problem is well known and can be written either analytically in terms of elliptic integrals or numerically. In the latter case, the solution was calculated by Gómez et al. ${ }^{11}$

Thus, the state variables can be perturbed as

$$
\begin{aligned}
& W(z, t)=\varepsilon w(z, \tau), \quad P(z, t)=P_{0}+\varepsilon p(z, \tau), \\
& F(z, t)=F_{0}(z)+\varepsilon f(z, \tau),
\end{aligned}
$$

where $w \sim p \sim f \sim 1$. Introducing the above expressions into Eqs. (2)-(4) and into the boundary conditions (6)-(8) leads to the following equations:

$$
\begin{aligned}
& p_{z}= \varepsilon\left\{-w_{\tau}-w w_{z}-\left(k_{1}+k_{2}\right)\left[-\frac{1}{2} F_{0} F_{0 z}\left(w_{\tau z}-\frac{1}{2} w_{z}^{2}+w w_{z z}\right)-\frac{1}{8} F_{0}{ }^{2}\left(w_{\tau z}-\frac{1}{2} w_{z}^{2}+w w_{z z}\right)\right]+c\left[3 w_{z z}+6 \frac{F_{0 z}}{F_{0}} w_{z}\right]\right. \\
&+c k_{1}\left[-\frac{1}{8} F_{0}{ }^{2} w_{z z z z}-F_{0} F_{0 z} w_{z z z}-\frac{1}{2}\left(F_{0} F_{0 z z}+3 F_{0 z}{ }^{2}\right) w_{z z}\right] \\
&\left.+\frac{3}{4} c k_{2}\left[\left(F_{0} F_{0 z z}-3 F_{0 z}{ }^{2}\right) w_{z z z}+\left(F_{0} F_{0 z z z}-3 F_{0 z} F_{0 z z}-6 \frac{F_{0 z}}{F_{0}} F_{0 z}{ }^{2}\right) w_{z z}\right]\right\}-\varepsilon^{2} b \\
&-\frac{1}{2} F_{0}(z) w_{z}(z, \tau)-F_{0 z}(z) w(z, \tau)=\varepsilon\left[f_{\tau}(z, \tau)+\frac{1}{2} f(z, \tau) w_{z}(z, \tau)+f_{z}(z, \tau) w(z, \tau)\right] \\
& \mathrm{M}\left[F_{0}(z)\right]+P_{0}=-\varepsilon\left\{\mathrm{M}^{\prime}\left[F_{0}(z)\right][f(z, \tau)]+p(z, \tau)\right\}+\varepsilon^{2}\left\{-\frac{1}{2} \mathrm{M}^{\prime \prime}\left[F_{0}(z)\right][f(z, \tau), f(z, \tau)]\right\} \\
&+\varepsilon^{3}\left\{-\frac{1}{6} \mathrm{M}^{\prime \prime \prime}\left[F_{0}(z)\right][f(z, \tau), f(z, \tau), f(z, \tau)]\right\}+O\left(\varepsilon^{4}\right),
\end{aligned}
$$

with boundary conditions

$$
\begin{aligned}
& w( \pm \Lambda, \tau)=0, \\
& w_{z}( \pm \Lambda, \tau)=0, \\
& F_{0}( \pm \Lambda)=1-\varepsilon f( \pm \Lambda, \tau) \pm \varepsilon^{3} h,
\end{aligned}
$$

and substitution of Eq. (19) into the volume preservation Eq. (9) yields

$$
\begin{aligned}
\int_{-\Lambda}^{\Lambda} F_{0}^{2}(z) \mathrm{d} z-2 \Lambda V_{0}= & -2 \varepsilon \int_{-\Lambda}^{\Lambda} F_{0}(z) f(z, \tau) \mathrm{d} z \\
& +\varepsilon^{2}\left\{-\int_{-\Lambda}^{\Lambda} f^{2}(z, \tau) \mathrm{d} z+2 \Lambda v\right\}
\end{aligned}
$$

Setting $\varepsilon=0$ in this formulation, we recover Eqs. (16)-(18) that provide the steady state,

At the first order, $O(\varepsilon)$, the following linear problem results:

$p_{z}=0$

$\mathrm{M}^{\prime}\left[F_{0}(z)\right][f(z, \tau)]+p(z, \tau)=0$, 


$$
\begin{aligned}
& f( \pm \Lambda, \tau)=0, \\
& \int_{-\Lambda}^{\Lambda} F_{0}(z) f(z, \tau) \mathrm{d} z=0 .
\end{aligned}
$$

Equation (27) means that $p$ does not depend on the axial coordinate $z$, namely, it is at most a function of time. Thus, $f$ and $p$ can be written as

$$
f(z, \tau)=a(\tau) f_{1}(z), \quad p(z, \tau)=a(\tau) p_{1},
$$

where, besides the above consideration, it has been taken into account that the operator $\mathrm{M}^{\prime}$ is linear and only contains spatial derivatives (but no temporal derivative). Thus, the problem that allows calculating $f_{1}$ and $p_{1}$ is

$$
\begin{aligned}
& \mathrm{M}^{\prime}\left[F_{0}(z)\right]\left[f_{1}(z)\right]+p_{1}=0 \\
& f_{1}( \pm \Lambda)=0 \\
& \int_{-\Lambda}^{\Lambda} F_{0}(z) f_{1}(z) \mathrm{d} z=0
\end{aligned}
$$

It is well known that this problem exhibits nontrivial solutions only for appropriate values $\Lambda$ and $V_{0}$, which precisely determines the lower instability limit in Fig. 2. The nontrivial solutions are antisymmetric in $z$. It follows that

$$
f_{1}(z)=F_{0 z}(z), \quad p_{1}=0,
$$

up to a constant factor. The amplitude $a(t)$ appearing in Eq. (31) remains unknown. It must satisfy an amplitude equation that will be derived below. Note that once the amplitude is calculated, Eq. (31) provides a first approximation of the evolution of both the interface shape and the pressure.

Taking into account the above results, it is seen that the appropriate expansions for the rescaled variables appearing in Eq. (19) which are to be introduced in Eqs. (20)-(26) are

$$
\begin{aligned}
& f(z, \tau)=a(\tau) f_{1}(z)+\varepsilon f_{2}(z, \tau)+\varepsilon^{2} f_{3}(z, \tau)+\cdots, \\
& p(z, \tau)=0+\varepsilon p_{2}(z, \tau)+\varepsilon^{2} p_{3}(z, \tau)+\cdots, \\
& w(z, \tau)=0+\varepsilon w_{2}(z, \tau)+\varepsilon^{2} w_{3}(z, \tau)+\cdots,
\end{aligned}
$$

where the leading order terms have been calculated above. Higher order (quadratic and cubic) corrections are now considered.

The first (quadratic) correction is calculated from

$$
\begin{aligned}
& p_{2 z}=0, \\
& w_{2}( \pm \Lambda, \tau)=0, \\
& w_{2 z}( \pm \Lambda, \tau)=0, \\
& -\frac{1}{2} F_{0}(z) w_{2 z}(z, \tau)-F_{0 z}(z) w_{2}(z, \tau)=\dot{a}(\tau) f_{1}(z), \\
& \mathbf{M}^{\prime}\left[F_{0}(z)\right]\left[f_{2}(z, \tau)\right]+p_{2}(z, \tau) \\
& \quad=-\frac{1}{2} a^{2}(\tau) \mathrm{M}^{\prime \prime}\left[F_{0}(z)\right]\left[f_{1}(z), f_{1}(z)\right],
\end{aligned}
$$

$f_{2}( \pm \Lambda, \tau)=0$,

$$
\int_{-\Lambda}^{\Lambda} F_{0}(z) f_{2}(z, \tau) \mathrm{d} z=-\frac{a^{2}(\tau)}{2} \int_{-\Lambda}^{\Lambda} f_{1}^{2}(z) \mathrm{d} z+\Lambda v .
$$

The second (cubic) correction is given by

$$
\begin{aligned}
& p_{3 z}=-w_{2 \tau}-\left(k_{1}+k_{2}\right)\left[-\frac{1}{2} F_{0} F_{0 z} w_{2 \tau z}-\frac{1}{8} F_{0}{ }^{2} w_{2 \tau z z}\right] \\
& +c\left[3 w_{2 z z}+6 \frac{F_{0 z}}{F_{0}} w_{2 z}\right]+c k_{1}\left[-\frac{1}{8} F_{0}{ }^{2} w_{2 z z z z}\right. \\
& \left.-F_{0} F_{0 z} w_{2 z z z}-\frac{1}{2}\left(F_{0} F_{0 z z}+3 F_{0 z}{ }^{2}\right) w_{2 z z}\right] \\
& +\frac{3}{4} c k_{2}\left[\left(F_{0} F_{0 z z}-3 F_{0 z}^{2}\right) w_{2 z z z}\right. \\
& \left.+\left(F_{0} F_{0 z z z}-3 F_{0 z} F_{0 z z}-6 \frac{F_{0 z}}{F_{0}} F_{0 z}^{2}\right) w_{2 z z}\right]-b, \\
& w_{3}( \pm \Lambda, \tau)=0 \text {, } \\
& w_{3 z}( \pm \Lambda, \tau)=0 \text {, } \\
& -\frac{1}{2} F_{0}(z) w_{3 z}(z, \tau)-F_{0 z}(z) w_{3}(z, \tau) \\
& =f_{2 \tau}(z, \tau)-a(\tau)\left[f_{1}(z) w_{2 z}(z, \tau)+f_{1 z}(z) w_{2}(z, \tau)\right] \text {, } \\
& \mathbf{M}^{\prime}\left[F_{0}(z)\right]\left[f_{3}(z, \tau)\right]+p_{3}(z, \tau) \\
& =-a(\tau) \mathrm{M}^{\prime \prime}\left[F_{0}(z)\right]\left[f_{1}(z), f_{2}(z, \tau)\right] \\
& -\frac{1}{6} a^{3}(\tau) \mathrm{M}^{\prime \prime \prime}\left[F_{0}(z)\right]\left[f_{1}(z), f_{1}(z), f_{1}(z)\right], \\
& f_{3}( \pm \Lambda, \tau)= \pm h, \\
& \int_{-\Lambda}^{\Lambda} F_{0}(z) f_{3}(z, \tau) \mathrm{d} z=-a(\tau) \int_{-\Lambda}^{\Lambda} f_{1}(z) f_{2}(z, \tau) \mathrm{d} z .
\end{aligned}
$$

The two problems above are now solved sequentially. Equation (36) implies that $p_{2}$ does not depend on $z$ (but only on $t$ ), that is $p_{2}=p_{2}(\tau)$. Thus, $f_{2}(z, \tau)$ and $p_{2}(\tau)$ are given by [cf. Eqs. (40)-(42)]

$$
\begin{aligned}
& \mathbf{M}^{\prime}\left[F_{0}(z)\right]\left[f_{2}(z, \tau)\right]+p_{2}(\tau) \\
& \quad=-\frac{1}{2} a^{2}(\tau) \mathrm{M}^{\prime \prime}\left[F_{0}(z)\right]\left[f_{1}(z), f_{1}(z)\right], \\
& f_{2}( \pm \Lambda, \tau)=0, \\
& \int_{-\Lambda}^{\Lambda} F_{0}(z) f_{2}(z, \tau) \mathrm{d} z=-\frac{a^{2}(\tau)}{2} \int_{-\Lambda}^{\Lambda} f_{1}^{2}(z) \mathrm{d} z+\Lambda v .
\end{aligned}
$$

As above, the linear character of the operator $\mathrm{M}^{\prime}$ allows splitting this problem into two simpler problems to determine $f_{2}$ and $p_{2}$, which is done setting

$$
\begin{aligned}
& f_{2}(z, \tau)=a^{2}(\tau) \tilde{f}_{11}(z)+v \tilde{f}_{2}(z), \\
& p_{2}(\tau)=a^{2}(\tau) \widetilde{p}_{11}+v \widetilde{p}_{2} .
\end{aligned}
$$

Substitution of these expressions into Eqs. (50)-(52) leads to the following problems to calculate $\left(\tilde{f}_{11}, \widetilde{p}_{11}\right)$ and $\left(\tilde{f}_{2}, \tilde{p}_{2}\right)$ : 
$\mathbf{M}^{\prime}\left[F_{0}(z)\right]\left[\tilde{f}_{11}(z)\right]+\widetilde{p}_{11}=-\frac{1}{2} \mathbf{M}^{\prime \prime}\left[F_{0}(z)\right]\left[f_{1}(z), f_{1}(z)\right]$,

$\tilde{f}_{11}( \pm \Lambda)=0$,

$\int_{-\Lambda}^{\Lambda} F_{0}(z) \tilde{f}_{11}(z) \mathrm{d} z=-\frac{1}{2} \int_{-\Lambda}^{\Lambda} f_{1}^{2}(z) \mathrm{d} z$,

and

$$
\begin{aligned}
& \mathrm{M}^{\prime}\left[F_{0}(z)\right]\left[\tilde{f}_{2}(z)\right]+\widetilde{p}_{2}=0, \\
& \tilde{f}_{2}( \pm \Lambda)=0 \\
& \int_{-\Lambda}^{\Lambda} F_{0}(z) \tilde{f}_{2}(z) \mathrm{d} z=\Lambda .
\end{aligned}
$$

Note that these two problems are singular, since their left hand sides coincide with the homogeneous linear problem (32)-(34), which exhibits nontrivial solutions. But the forcing terms are reflection symmetric in $z$, while nontrivial solutions of homogeneous part are antisymmetric, which means that the problems above are solvable. The resulting solutions are of course nonunique since any solution of the homogeneous problem can be added to a given solution. But the amplitude equation that will be derived below is independent of the particular solution that is selected. For convenience, we select the particular solution such that

$$
\begin{aligned}
& \int_{-\Lambda}^{\Lambda} F_{0}(z) f_{1}(z) \tilde{f}_{11}(z) \mathrm{d} z=0, \\
& \int_{-\Lambda}^{\Lambda} F_{0}(z) f_{1}(z) \tilde{f}_{2}(z) \mathrm{d} z=0 .
\end{aligned}
$$

Note that in the two problems above, $f_{1}(z)$ is a known function [it is equal to $F_{0 z}(z)$ ]. These problems are closely related to those appearing in the study of the statics of liquid bridges and have been already solved analytically (in terms of elliptic integrals) by Gómez et al. ${ }^{11}$ It is useful to note that neither $\widetilde{p}_{11}$ nor $\widetilde{p}_{2}$ are needed to determine the dynamical behavior. Instead, only $\tilde{f}_{11}(z)$ and $\tilde{f}_{2}(z)$ need to be computed.

Once that the above problems have been solved, a substitution into the remaining equations (taking into account that $p_{2}$ depends only on $\tau$ ) yields

$$
\begin{aligned}
w_{2 \tau}+ & \left(k_{1}+k_{2}\right)\left[-\frac{1}{2} F_{0} F_{0 z} w_{2 \pi z}-\frac{1}{8} F_{0}{ }^{2} w_{2 \pi z z}\right] \\
= & -\bar{p}_{3 z}+c\left[3 w_{2 z z}+6 \frac{F_{0 z}}{F_{0}} w_{2 z}\right] \\
& +c k_{1}\left[-\frac{1}{8} F_{0}{ }^{2} w_{2 z z z z}-F_{0} F_{0 z} w_{2 z z z}-\frac{1}{2}\left(F_{0} F_{0 z z}\right.\right. \\
& \left.\left.+3 F_{0 z}{ }^{2}\right) w_{2 z z}\right]+\frac{3}{4} c k_{2}\left[\left(F_{0} F_{0 z z}-3 F_{0 z}{ }^{2}\right) w_{2 z z z}\right. \\
& \left.+\left(F_{0} F_{0 z z z}-3 F_{0 z} F_{0 z z}-6 \frac{F_{0 z}}{F_{0}} F_{0 z}{ }^{2}\right) w_{2 z z}\right],
\end{aligned}
$$

$$
\begin{aligned}
& w_{2}( \pm \Lambda, \tau)=0, \\
& w_{2 z}( \pm \Lambda, \tau)=0, \\
& -\frac{1}{2} F_{0}(z) w_{2 z}(z, \tau)-F_{0 z}(z) w_{2}(z, \tau)=\dot{a}(\tau) f_{1}(z), \\
& \mathbf{M}^{\prime}\left[F_{0}(z)\right]\left[f_{3}(z, \tau)\right]+\bar{p}_{3}(z, \tau)-b z \\
& =-a(\tau) v \mathbf{M}^{\prime \prime}\left[F_{0}(z)\right]\left[f_{1}(z), \tilde{f}_{2}(z)\right] \\
& -a^{3}(\tau) \mathrm{M}^{\prime \prime}\left[F_{0}(z)\right]\left[f_{1}(z), \tilde{f}_{11}(z)\right] \\
& -\frac{1}{6} a^{3}(\tau) \mathbf{M}^{\prime \prime \prime}\left[F_{0}(z)\right]\left[f_{1}(z), f_{1}(z), f_{1}(z)\right], \\
& f_{3}( \pm \Lambda, \tau)= \pm h, \\
& \int_{-\Lambda}^{\Lambda} F_{0}(z) f_{3}(z, \tau) \mathrm{d} z=-a(\tau) v \int_{-\Lambda}^{\Lambda} f_{1}(z) \tilde{f}_{2}(z) \mathrm{d} z \\
& -a^{3}(\tau) \int_{-\Lambda}^{\Lambda} f_{1}(z) \tilde{f}_{11}(z) \mathrm{d} z
\end{aligned}
$$

where for convenience, we have set

$$
p_{3}(z, \tau)=\bar{p}_{3}(z, \tau)-b z .
$$

The equations above suggest rewriting the unknowns $\bar{p}_{3}$ and $w_{2}$ as

$$
\bar{p}_{3}(z, \tau)=\ddot{a}(\tau) \Pi_{2}(z)+c \dot{a}(\tau) \Pi_{1}(z), \quad w_{2}(z, \tau)=\dot{a}(\tau) \Omega_{2}(z) .
$$

Substitution of the latter expression into Eqs. (62)-(64) yields

$$
\begin{aligned}
& -\frac{1}{2} F_{0}(z) \Omega_{2 z}(z)-F_{0 z}(z) \Omega_{2}(z)=f_{1}(z), \\
& \Omega_{2}( \pm \Lambda)=0, \\
& \Omega_{2 z}( \pm \Lambda)=0 .
\end{aligned}
$$

Note that $\Omega_{2}$ is a symmetric function of $z$ (as $F_{0}$ is), while $f_{1}$ and $F_{0 z}$ are antisymmetric. Thus, the conditions (69) and (70) are redundant and it suffices to integrate Eq. (68) starting with $\Omega_{2}(-\Lambda)=0$ to obtain $\Omega_{2}$. Substituting $\Omega_{2}$ into Eq. (61) leads to the following equations for $\Pi_{1}$ and $\Pi_{2}$ :

$$
\begin{aligned}
0= & -\Pi_{1 z}+\left[3 \Omega_{2 z z}+6 \frac{F_{0 z}}{F_{0}} \Omega_{2 z}\right] \\
& +k_{1}\left[-\frac{1}{8} F_{0}{ }^{2} \Omega_{2 z z z z}-F_{0} F_{0 z} \Omega_{2 z z z}-\frac{1}{2}\left(F_{0} F_{0 z z}\right.\right. \\
& \left.\left.+3 F_{0 z}{ }^{2}\right) \Omega_{2 z z}\right]+\frac{3}{4} k_{2}\left[\left(F_{0} F_{0 z z}-3 F_{0 z}{ }^{2}\right) \Omega_{2 z z z}\right. \\
& \left.+\left(F_{0} F_{0 z z z}-3 F_{0 z} F_{0 z z}-6 \frac{F_{0 z}}{F_{0}} F_{0 z}{ }^{2}\right) \Omega_{2 z z}\right]
\end{aligned}
$$

and

$$
\Omega_{2}+\left(k_{1}+k_{2}\right)\left[-\frac{1}{2} F_{0} F_{0 z} \Omega_{2 z}-\frac{1}{8} F_{0}{ }^{2} \Omega_{2 z z}\right]=-\Pi_{2 z} .
$$

These equations determine $\Pi_{1}$ and $\Pi_{2}$ up to a translation constant, which is not relevant to the final result below. For 
convenience, $\Pi_{1}$ and $\Pi_{2}$ will be computed as

$$
\Pi_{1}(z)=\Pi_{10}(z)+k_{1} \Pi_{11}(z)+k_{2} \Pi_{12}(z)
$$

and

$$
\Pi_{2}(z)=\Pi_{20}(z)+\left(k_{1}+k_{2}\right) \Pi_{21}(z) .
$$

Once the above problems have been solved and the values of $\Pi_{1}$ and $\Pi_{2}$ are known, a further substitution into Eq. (65) yields the following two-point boundary value problem to determine $f_{3}$ :

$$
\begin{aligned}
& \mathrm{M}^{\prime}\left[F_{0}(z)\right]\left[f_{3}(z, \tau)\right]+\ddot{a}(\tau) \Pi_{2}(z)+\dot{a}(\tau) \Pi_{1}(z)-b z \\
&=-a(\tau) v \mathrm{M}^{\prime \prime}\left[F_{0}(z)\right]\left[f_{1}(z), \tilde{f}_{2}(z)\right] \\
&-a^{3}(\tau) \mathrm{M}^{\prime \prime}\left[F_{0}(z)\right]\left[f_{1}(z), \tilde{f}_{11}(z)\right] \\
&-\frac{1}{6} a^{3}(\tau) \mathrm{M}^{\prime \prime \prime}\left[F_{0}(z)\right]\left[f_{1}(z), f_{1}(z), f_{1}(z)\right],
\end{aligned}
$$

$$
f_{3}( \pm \Lambda, \tau)= \pm h,
$$

$$
\begin{aligned}
\int_{-\Lambda}^{\Lambda} F_{0}(z) f_{3}(z, \tau) \mathrm{d} z= & -a(\tau) v \int_{-\Lambda}^{\Lambda} f_{1}(z) \tilde{f}_{2}(z) \mathrm{d} z \\
& -a^{3}(\tau) \int_{-\Lambda}^{\Lambda} f_{1}(z) \tilde{f}_{11}(z) \mathrm{d} z
\end{aligned}
$$

$$
\int_{-\Lambda}^{\Lambda} F_{0}(z) f_{1}(z) f_{3}(z, \tau) \mathrm{d} z=0 .
$$

It is to be noted that this problem need not be fully solved to obtain the amplitude equation, which is the final object of this section. Instead, we only need to apply a solvability condition, noting that the homogeneous part of Eqs. (75)-(78) exhibits nontrivial solutions [cf. Eqs. (32)-(34) above]. Such solvability condition is readily obtained multiplying Eq. (75) by $F_{0}(z) f_{1}(z)$ and integrating the resulting equation in $-\Lambda \leq z \leq \Lambda$ to obtain

$$
\begin{aligned}
\int_{-\Lambda}^{\Lambda} \mathrm{M}^{\prime}\left[F_{0}(z)\right]\left[f_{3}(z, \tau)\right] F_{0}(z) f_{1}(z) \mathrm{d} z+\ddot{a}(\tau) \int_{-\Lambda}^{\Lambda} \Pi_{2}(z) F_{0}(z) f_{1}(z) \mathrm{d} z+\dot{a}(\tau) c \int_{-\Lambda}^{\Lambda} \Pi_{1}(z) F_{0}(z) f_{1}(z) \mathrm{d} z-b \int_{-\Lambda}^{\Lambda} z F_{0}(z) f_{1}(z) \mathrm{d} z \\
=-a(\tau) v \int_{-\Lambda}^{\Lambda} \mathbf{M}^{\prime \prime}\left[F_{0}(z)\right]\left[f_{1}(z), \tilde{f}_{2}(z)\right] F_{0}(z) f_{1}(z) \mathrm{d} z \\
\quad-a^{3}(\tau) \int_{-\Lambda}^{\Lambda}\left\{\mathbf{M}^{\prime \prime}\left[F_{0}(z)\right]\left[f_{1}(z), \tilde{f}_{11}(z)\right]+\frac{1}{6} \mathbf{M}^{\prime \prime \prime}\left[F_{0}(z)\right]\left[f_{1}(z), f_{1}(z), f_{1}(z)\right]\right\} F_{0}(z) f_{1}(z) \mathrm{d} z,
\end{aligned}
$$

where the unknown $f_{3}$ is still present in the first integral. But this can be eliminated using the following expression, which is obtained integrating by parts,

$$
\begin{aligned}
& \int_{-\Lambda}^{\Lambda} \mathrm{M}^{\prime}\left[F_{0}(z)\right][g(z)] F_{0}(z) f_{1}(z) \mathrm{d} z \\
& \quad=-\left.\frac{F_{0}(z) f_{1 z}(z) g(z)}{\left[1+F_{0 z}^{2}(z)\right]^{3 / 2}}\right|_{-\Lambda} ^{\Lambda}-p_{1} \int_{-\Lambda}^{\Lambda} F_{0}(z) g(z) \mathrm{d} z
\end{aligned}
$$

Here, $g$ is any smooth enough function. Setting $g=f_{3}$ yields, using boundary conditions (66), and the fact that $p_{1}=0$, we obtained that

$$
\int_{-\Lambda}^{\Lambda} \mathrm{M}^{\prime}\left[F_{0}(z)\right]\left[f_{3}(z, \tau)\right] F_{0}(z) f_{1}(z) \mathrm{d} z=-2 h \frac{F_{0}(\Lambda) f_{1 z}(\Lambda)}{\left[1+F_{0 z}^{2}(\Lambda)\right]^{3 / 2}} .
$$

Using all these, Eq. (79) provides the following equation for the unknown amplitude $a(\tau)$ :

$$
\mu \ddot{a}(\tau)+\gamma c \dot{a}(\tau)+\widetilde{g}_{12} v a(\tau)+\widetilde{g}_{111} a^{3}(\tau)=\widetilde{g}_{3} h+\widetilde{g}_{4} b,
$$

where

$$
\mu=\mu_{0}+\left(k_{1}+k_{2}\right) \mu_{1}, \quad \gamma=\gamma_{0}+k_{1} \gamma_{1}+k_{2} \gamma_{2},
$$

and

$$
\begin{aligned}
& \mu_{0}=\int_{-\Lambda}^{\Lambda} \Pi_{20}(z) F_{0}(z) f_{1}(z) \mathrm{d} z, \\
& \mu_{1}=\int_{-\Lambda}^{\Lambda} \Pi_{21}(z) F_{0}(z) f_{1}(z) \mathrm{d} z,
\end{aligned}
$$

$\gamma_{0}=\int_{-\Lambda}^{\Lambda} \Pi_{10}(z) F_{0}(z) f_{1}(z) \mathrm{d} z$

$$
\gamma_{1}=\int_{-\Lambda}^{\Lambda} \Pi_{11}(z) F_{0}(z) f_{1}(z) \mathrm{d} z
$$

$$
\gamma_{2}=\int_{-\Lambda}^{\Lambda} \Pi_{12}(z) F_{0}(z) f_{1}(z) \mathrm{d} z \equiv 0,
$$

$$
\tilde{g}_{12}=\int_{-\Lambda}^{\Lambda} \mathrm{M}^{\prime \prime}\left[F_{0}(z)\right]\left[f_{1}, \tilde{f}_{2}\right] F_{0}(z) f_{1}(z) \mathrm{d} z
$$




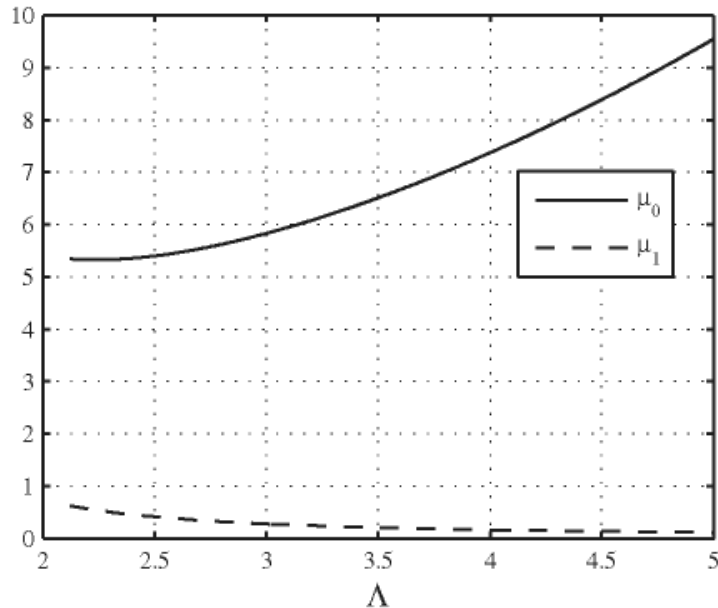

FIG. 3. Mass coefficients for the antisymmetric mode, $\mu_{0}$ and $\mu_{1}$.

$$
\begin{aligned}
& \widetilde{g}_{111}= \int_{-\Lambda}^{\Lambda}\left\{\mathrm{M}^{\prime \prime}\left[F_{0}(z)\right]\left[f_{1}, \tilde{f}_{11}\right]\right. \\
&\left.+\frac{1}{6} \mathrm{M}^{\prime \prime \prime}\left[F_{0}(z)\right]\left[f_{1}, f_{1}, f_{1}\right]\right\} F_{0}(z) f_{1}(z) \mathrm{d} z, \\
& \widetilde{g}_{3}= 2 \frac{F_{0}(\Lambda) f_{1 z}(\Lambda)}{\left[1+F_{0 z}^{2}(\Lambda)\right]^{3 / 2}}, \\
& \widetilde{g}_{4}=\int_{-\Lambda}^{\Lambda} z F_{0}(z) f_{1}(z) \mathrm{d} z .
\end{aligned}
$$

The mass coefficient values $\mu_{0}$ and $\mu_{1}$, the damping coefficients $\gamma_{0}$ and $\gamma_{1}$, and the stability coefficient values are shown in Figs. 3-5, as calculated from Eqs. (84)-(92). The coefficient $\gamma_{2}$ identically vanishes, see Eq. (88). This is because due to the symmetries of $F_{0}$ and $\Omega_{2}, \Pi_{12}$ is a symmetric function of $z$, as $F_{0}$ is, while $f_{1}$ is antisymmetric. The plots of the remaining coefficients show that (i) the cubic coefficient $\widetilde{g}_{111}$ is always negative (Fig. 5), which is associated with the fact that the instability is subcritical. Also, (ii) $\mu_{1}$ and $\gamma_{1}$ are much smaller (between 10 and 20 times smaller) than $\mu_{0}$ and $\gamma_{0}$ (see Figs. 3 and 4). This means invoking Eq. (83) that both the mass and damping coefficients depend only weakly on $k_{1}$ and $k_{2}$. Since these two

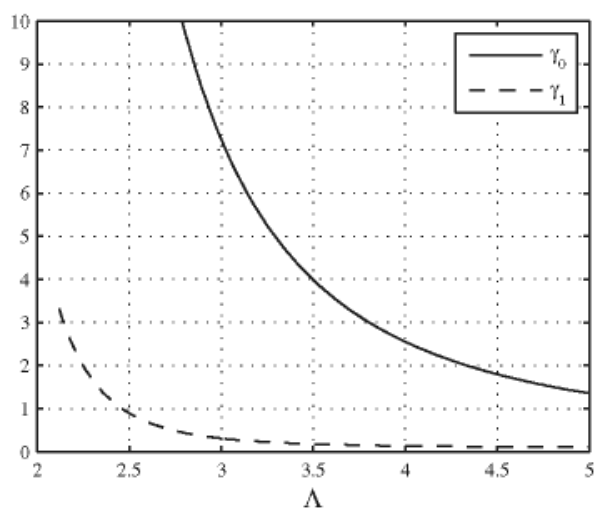

FIG. 4. Damping coefficients for the antisymmetric mode, $\gamma_{0}$ and $\gamma_{1}$.

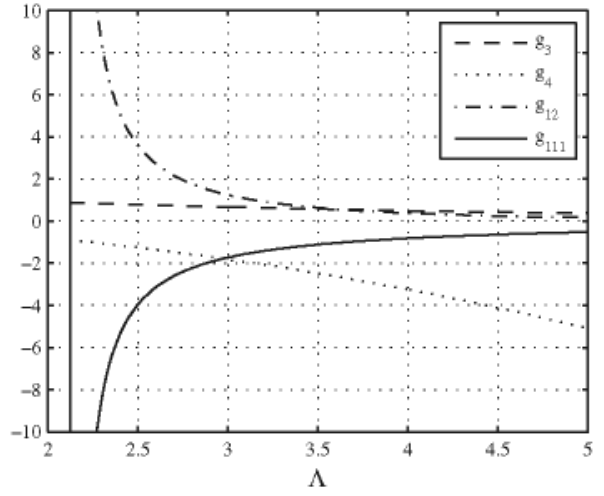

FIG. 5. Stability coefficients. The different coefficients have been calculated for $\Lambda>\Lambda_{A}$, shown by a vertical line in the figure.

latter adjustable coefficients account for the particular onedimensional model that is being used, the conclusion is that the amplitude Eq. (82) is fairly independent of the onedimensional model.

As a third conclusion, (iii) for slender liquid bridges, the mass and damping coefficients $\mu \simeq \mu_{0}$ and $\gamma \simeq \gamma_{0}$ are both much larger than the stability coefficients $\widetilde{g}_{12}, \widetilde{g}_{111}, \widetilde{g}_{3}$, and $\tilde{g}_{4}$, which are all comparable. As the slenderness decreases, $\widetilde{g}_{12}, \widetilde{g}_{111}$, and $\gamma$ become dominant, which means in particular that damping and cubic nonlinearity are the most important effects, but this is an artifact of the one-dimensional approximation, which only applies for slender configurations.

In the original dimensionless variables, without rescaling and defining $A(t)=\varepsilon a(\tau)$, the perturbation of the interface shape is given by

$$
F(z, t)=F_{0}(z)+A(t) f_{1}(z),
$$

with $F_{0}(z)$ and $f_{1}(z)$ as given by the solutions of the problem above. The amplitude equation, Eq. (82), is rewritten as

$$
\mu \frac{\mathrm{d}^{2} A(t)}{\mathrm{d} t^{2}}+\gamma c \frac{\mathrm{d} A(t)}{\mathrm{d} t}+\widetilde{g}_{12} V A(t)+\widetilde{g}_{111} A^{3}(t)=\widetilde{g}_{3} H+\widetilde{g}_{4} B,
$$

which is a standard Duffing equation. In the above equation, $V, H$, and/or $B$ may be slowly varying with time (although, for most applications, only $B$ will be considered as variable). The various mass, damping, and stability coefficients are only functions of $\Lambda$.

Equation (94) shows that the volume perturbation $V$ does not break the reflection symmetry on the midplane [which corresponds to the action $A \rightarrow-A$ in Eq. (94)] and since $\widetilde{g}_{12}$ $>0$ (see Fig. 5) has a stabilizing and a destabilizing effect if $V>0$ and $V<0$, respectively, which is in accordance with the fact that the liquid bridge is stable if the volume is larger than the lower instability limit in Fig. 2. The remaining perturbations (inequality of the disk radii and gravity) instead break reflection symmetry and promote destabilization, irrespective of the signs of $H$ and $B$. This is because these two effects break the subcritical pitchfork bifurcation that applies when $B=H=0$; see, e.g., Ref. 10 for an early discussion of the role of reflection symmetries in perturbed instabilities of liquid bridge configurations. 

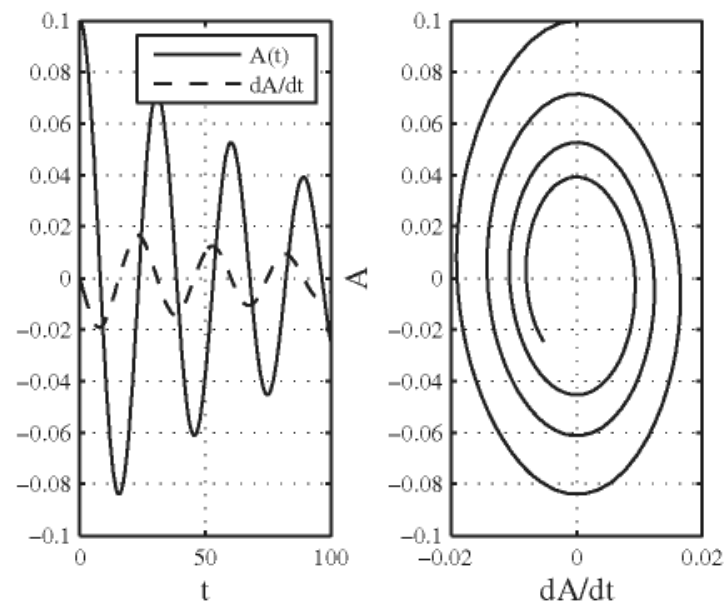

FIG. 6. Time evolution of the amplitude and its derivative with time for a stable configuration $(\Lambda=\pi, V=+0.05, C=0.02, H=B=0)$. Cosserat model has been used, $k_{1}=1, k_{2}=0$.

As an example of the application of the amplitude equation, Figs. 6 and 7 show the evolution of the amplitude and its derivative with time for a stable configuration $(\Lambda=\pi, V=+0.05, C=0.02, H=B=0)$ and an unstable configuration $(\Lambda=\pi, V=-0.05, C=0.02, H=B=0)$. Initial conditions are the same for both cases, $A(0)=0.1$, $\mathrm{d} A / \mathrm{d} t(0)=0$. As can be seen, a nonlinear oscillation results for large time in the stable case (Fig. 6), which exhibits quite a large period. In the unstable case instead, the oscillation blows up. The latter can be seen as a first approximation of the breakage time in this case, even though Eq. (94) only describes the first stage of breakage (while $|A|$ remains small), which occurs in a quite slow characteristic time, $t \sim 1 /|A|$; the subsequent stage involves nonsmall perturbations of the shape of the interface, and thus occurs in a much faster time, $t \sim 1$.
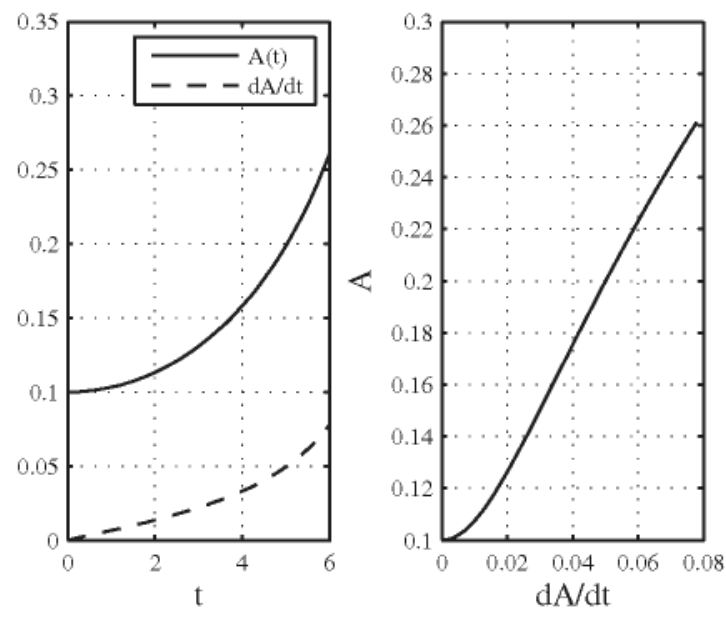

FIG. 7. Time evolution of the amplitude and its derivative with time for an unstable configuration $(\Lambda=\pi, V=-0.05, C=0.02, H=B=0)$. Cosserat model has been used, $k_{1}=1, k_{2}=0$.

\section{CONCLUSIONS}

An amplitude equation has been derived that provides, in a simple way, the marginally unstable interface behavior of a liquid bridge when this is close to the steady state. The stability limit considered has been that of minimum volume, where two different types of instability may appear, one leading to reflection symmetric deformations (symmetric breakages) with regard to $z$ for $\Lambda_{C}<\Lambda<\Lambda_{A}$, and the other leading to reflection antisymmetric deformations (antisymmetric breakages) with regard to $z$ for $\Lambda>\Lambda_{A}$. Only the latter case has been considered as the model for the flow dynamics is one-dimensional and this model is only accurate for slender enough liquid bridges. A general one-dimensional dynamics model has been considered that includes as particular cases the Lee, Cosserat, and averaged models and allows for the easy consideration of the effects of the viscosity in the bulk, which is the main effect in this case, since the anchoring of the interface to the edges of the disks leads to a velocity field where the dissipation in the boundary layers is very weak. This is because the radial velocity field compatible with onedimensional models is zero at the disks and there is no need for a strong boundary layer to fulfill the zero radial velocity boundary condition. The model also allows including the effects of the small change of volume, disk unequality, and axial gravity, either stationary or slowly varying with time. The amplitude equation that governs the weakly nonlinear dynamics has been seen to be fairly independent of the details of the one-dimensional model, which is good news in connection with the generality of the conclusions of the paper.

The Duffing-like amplitude equation derived in this paper is very simple. The time integration of the equation (with its coefficients already known for a given configuration) allows predicting not only the stability or instability of the configuration but the weakly nonlinear behavior of the configuration, close to the instability limit. This provides either the resulting nonlinear oscillations (in the stable side) or the time. We hope that this equation will be useful in the fast and easy prediction of the nearly unstable liquid bridge dynamics.

${ }^{1}$ J. Meseguer, J. M. Perales, I. Martínez, N. A. Bezdenejnykh, and A. Sanz, "Hydrostatic instabilities in floating zone crystal growth process," Curr. Top. Cryst. Growth Res. 5, 27 (1999).

${ }^{2}$ L. Rayleigh, "On the instability of jets," Proc. London Math. Soc. s1-10, 4 (1878).

${ }^{3}$ L. A. Slobozhanin and J. M. Perales, "Stability of liquid bridges between equal disks in an axial gravity field," Phys. Fluids A 5, 1305 (1993).

${ }^{4}$ L. A. Slobozhanin, M. Gómez, and M. J. M. Perales, "Stability of liquid bridges between unequal disks under zero-gravity conditions," Microgravity Sci. Technol. 8, 23 (1995).

${ }^{5}$ L. A. Slobozhanin and J. M. Perales, "Stability of isorotating liquid bridges between equal disks under zero-gravity conditions," Phys. Fluids 8, 2307 (1996).

${ }^{6}$ L. A. Slobozhanin and J. I. D. Alexander, "Stability of an isorotating liquid bridge in an axial gravity field," Phys. Fluids 9, 1880 (1997).

${ }^{7}$ L. A. Slobozhanin and J. I. D. Alexander, "Combined effect of disk inequality and axial gravity on axisymmetric liquid bridge stability," Phys. Fluids 10, 2473 (1998).

${ }^{8}$ A. Laverón-Simavilla and J. M. Perales, "Equilibrium shapes of nonaxisymmetric liquid bridges of arbitrary volume in gravitational fields and their potential energy," Phys. Fluids 7, 1204 (1995).

${ }^{9}$ A. Laverón-Simavilla and E. Checa, "Effect of a lateral gravitational field 
on the nonaxisymmetric equilibrium shapes of liquid bridges held between eccentric disks and of volumes equal to those of cylinders," Phys. Fluids 9, 817 (1997)

${ }^{10}$ J. M. Vega and J. M. Perales, "Almost cylindrical isorotating liquid bridges for small Bond numbers," Report No. ESA SP-191, 1983.

${ }^{11}$ M. Gómez, I. Parra, and J. M. Perales, "Mechanical imperfections effect on the minimum stability limit of liquid bridges," Phys. Fluids 14, 2029 (2002).

${ }^{12}$ J. M. Perales, J. Meseguer, and I. Martínez, "Minimum volume of axisymmetric liquid bridges between unequal disks in an axial microgravity field," J. Cryst. Growth 110, 855 (1991).

${ }^{13}$ N. A. Bezdenejnykh, J. Meseguer, and J. M. Perales, "Experimental analysis of stability limits of capillary liquid bridges," Phys. Fluids A 4, 677 (1992).

${ }^{14}$ N. A. Bezdenejnykh, J. Meseguer, and J. M. Perales, "An experimental analysis of the instability of non-axisymmetric liquid bridges in a gravitational field," Phys. Fluids 11, 3181 (1999).

${ }^{15} \mathrm{~J}$. A. Nicolás, "Frequency response of axisymmetric liquid bridges to an oscillatory microgravity field," Microgravity Sci. Technol. IV, 188 (1991).

${ }^{16}$ D. Langbein, "Oscillations of finite liquid columns," Microgravity Sci. Technol. V, 73 (1992).

${ }^{17}$ Y. Zhang and J. I. D. Alexander, "Sensitivity of liquid bridges subject to axial residual acceleration," Phys. Fluids A 2, 1966 (1990).

${ }^{18}$ J. M. Perales and J. Meseguer, "Theoretical and experimental study of the vibration of axisymmetric viscous liquid bridges," Phys. Fluids A 4, 1110 (1992).

${ }^{19} \mathrm{~J}$. A. Nicolás and J. M. Vega, "Linear oscillations of axisymmetric viscous liquid bridges," Z. Angew. Math. Phys. 51, 701 (2000).

${ }^{20}$ J. M. Montanero, "Theoretical analysis of the vibration of axisymmetric liquid bridges of arbitrary shape," Theor. Comput. Fluid Dyn. 16, 171 (2003).

${ }^{21}$ J. M. Montanero, "Linear dynamics of axisymmetric liquid bridges," Eur. J. Mech. B/Fluids 22, 167 (2003).

${ }^{22}$ A. Gañán and A. Barrero, "Free oscillations of liquid captive drops," Microgravity Sci. Technol. III, 70 (1990).

${ }^{23}$ J. A. Nicolás, D. Rivas, and J. M. Vega, "On the steady streaming flow due to high-frequency vibration in nearly inviscid liquid bridges," J. Fluid Mech. 354, 147 (1998).

${ }^{24}$ I. Martínez, J. M. Perales, and J. Meseguer, "Non-linear response of a liquid bridge to a sinusoidal acceleration under microgravity," Exp. Fluids 37, 775 (2004).

${ }^{25}$ I. Martínez, J. Meseguer, and J. M. Perales, "Wobbling of a liquid column between unequal discs," Adv. Space Res. 36, 26 (2005).

${ }^{26}$ R. M. S. M. Schulkes, "Nonlinear dynamics of liquid columns: A comparative study," Phys. Fluids A 5, 2121 (1993).

${ }^{27} \mathrm{~J}$. Eggers, "Nonlinear dynamics and breakup of free-surface flows," Rev. Mod. Phys. 69, 865 (1997).
${ }^{28} \mathrm{~T}$. Chen and J. Tsamopoulos, "Nonlinear dynamics of capillary liquid bridges: Theory," J. Fluid Mech. 255, 373 (1993).

${ }^{29}$ D. J. Mollot, J. Tsamopoulos, T. Chen, and N. Ashgriz, "Nonlinear dynamics of capillary liquid bridges: Experiments," J. Fluid Mech. 255, 411 (1993).

${ }^{30}$ S. Ahrens, F. Falk, R. Großbach, and D. Langbein, "Experiments on oscillations of small liquid bridges," Microgravity Sci. Technol. VII, 2 (1994).

${ }^{31}$ C. Ferrera, M. G. Cabezas, and J. M. Montanero, "An experimental analysis of the linear vibration of axisymmetric liquid bridges," Phys. Fluids 18, 082105 (2006).

${ }^{32} \mathrm{E}$. J. Vega and J. M. Montanero, "Damping of linear oscillations in axisymmetric liquid bridges," Phys. Fluids 21, 092101 (2009).

${ }^{33} \mathrm{~A}$. Sanz, "The influence of the outer bath in the dynamics of axisymmetric liquid bridges," J. Fluid Mech. 156, 101 (1985).

${ }^{34}$ M. Higuera, J. A. Nicolás, and J. M. Vega, "Linear oscillations of weakly dissipative axisymmetric liquid bridges," Phys. Fluids 6, 438 (1994).

${ }^{35}$ M. Higuera, J. A. Nicolas, and J. M. Vega, "Weakly nonlinear nonaxisymmetric oscillations of capillary bridges at small viscosity," Phys. Fluids 14. 3251 (2002)

${ }^{36} \mathrm{~J}$. A. Nicolás and J. M. Vega, "Weakly nonlinear oscillations of nearly inviscid axisymmetric liquid bridges," J. Fluid Mech. 328, 95 (1996).

${ }^{37} \mathrm{D}$. Rivas and J. Meseguer, "One-dimensional self-similar solution of the dynamics of axisymmetric slender liquid bridges," J. Fluid Mech. 138, 417 (1984).

${ }^{38}$ F. J. García and A. Castellanos, "One-dimensional models for slender axisymmetric viscous liquid jets," Phys. Fluids 6, 2676 (1994).

${ }^{39} \mathrm{~F}$. J. García and A. Castellanos, "One-dimensional models for slender axisymmetric viscous liquid bridges," Phys. Fluids 8, 2837 (1996).

${ }^{40}$ J. M. Montanero and F. J. Acero, "A note on the use of the onedimensional models to describe the linear dynamics of liquid bridges," Eur. J. Mech. B/Fluids 24, 288 (2005).

${ }^{41}$ J. Meseguer, M. A. González, and J. I. D. Alexander, "Dynamic stability of long, axisymmetric liquid bridges," Microgravity Sci. Technol. VII, 246 (1994)

${ }^{42}$ C. Martel, J. A. Nicolás, and J. M. Vega, "Surface-wave damping in a brimful circular cylinder," J. Fluid Mech. 360, 213 (1998).

${ }^{43}$ I. Martínez and J. M. Perales, "Liquid bridge stability data," J. Cryst. Growth 78, 369 (1986).

${ }^{44}$ A. H. Nayfeh and D. T. Mook, Nonlinear Oscillations (John Wiley, New York, 1979).

${ }^{45} \mathrm{~J}$. Guckenheimer and P. Holmes, Nonlinear Oscillations, Dynamical Systems, and Bifurcations of Vector Fields (Springer-Verlag, New York, 1983).

${ }^{46}$ F. C. Moon, Chaotic Vibrations: An Introduction for Applied Scientists and Engineers (John Wiley, New York, 1987).

${ }^{47}$ J. M. T. Thompson and H. B. Stewart, Nonlinear Dynamics and Chaos (John Wiley, Chichester, 2002). 